\title{
Inhalt des Jahrgangs 40 Sommaire du volume 40
}

Heinz Balmer, Zu Besuch bei einer Enkelin Felix Mendelssohns $\quad 15$

Luigi Belloni, Der Beitrag von Francesco Buzzi zur Entdeckung der «Macula lutea» und der «Fovea centralis» des menschlichen Auges

Urs Boschung, Iatromechanik und Chirurgie bei Lorenz Heister

Urs Boschung/Beat Rüttimann, Herrn Professor Dr. med. H. M. Koelbing zum 60. Geburtstag W.F.Bynum, Darwin and the Doctors: Evolution, Diathesis and Germs in 19 th Century Britain

Adolf Faller, Welchen Platz nimmt Stensens anatomische Forschung in Lorenz Heisters Chirurgie und Anatomie ein?

Hans-Rudolf Fehlmann, Beziehungen zwischen Arzt und Apotheker im 16. bis 18. Jahrhundert in der Schweiz

Esther Fischer-Homberger, Die gerichtsmedizinische Wundbegutachtung vom 16. bis 18. Jahrhundert

H.P. Hartmann, Medizinstudium und ärztliche Ethik

Huldrych M. Koelbing, Bemerkungen zu Ingrid Rohlands und Gundolf Keils "Randnotizen zum «Schüpfheimer Kodex»»

Huldrych M. Koelbing, Verzeichnis der Veröffentlichungen

Fridolf Kudlien, Schaustellerei und Heilmittelvertrieb in der Antike

Erna Lesky, Theorie und Praxis, aufgezeigt an den Wiener geburtshilflichen Lehrkanzeln 1752-1859

Nikolaus Mani, Neue Konzepte der Pathologie im 17. Jahrhundert

Markus Mattmüller, Die Pest in Liestal. Notizen zu den demographischen Implikationen der frühneuzeitlichen Epidemien

Marta Meyer-Salzmann, Aus der medizinischen Vergangenheit in Langenthal

W.E. Knowles Middleton, The Sun glasses used by H. B. de Saussure

Marie-Louise Portmann, Der Basler Medizinprofessor Johann Jacob Harder (1656-1711) als Geburtshelfer

Loris Premuda, Die Medizin in der Sicht eines italienischen Denkers der Aufklärung

Friedrich Rintelen, Der Basler Chirurg und Rebell Johannes Fatio (1649-1691)

Ingrid Rohland und Gundolf Keil, Randnotizen zum «Schüpfheimer Kodex〉

Gerhard Rudolph, Herkunft und Schicksal des Humor aqueus

Beat Rüttimann, Hippokratische «Wirbelverrenkung»

Hans-Konrad Schmutz, Die Gründung des Zürcher Lehrstuhles für Anthropologie

Heinrich Stamm, 50 Jahre medizinische Forschung in Waldenburg

Jean Starobinski, D'Agrippa de Nettesheim à Montaigne: L'embarras des médecins devant l'origine de la semence

Antoinette Stettler, Vorläufige medizinhistorische und pathographische Bemerkungen zu Ulrich Bräker

Jean Théodoridès, Ernst Jünger ou «l'œil vivant»

Ulrich Tröhler, Theodor Kocher und die neurotopographische Diagnostik:

Angewandte Forschung mit grundlegendem Ergebnis um 1900 
Théodor Vetter, Témoignages due Strasbourgeois Victor Stoeber sur la médecine parisienne (1824-1825)

Vera Waldis, Der «Stich» von 1564-eine primäre Lungenpest

Buchbesprechungen

Beiträge zur Schwäbischen Literatur- und Geistesgeschichte Bd.2/1982 (Carl Haffter)

Alexander Berghaus, Die Entwicklung der Methoden zur physiologischen Wertbestimmung von Digitalispräparaten (Erwin E. Ackerknecht)

L. Boulle, M.D.Grmek, C. Lupovici, J.Samion-Contet, Laennec. Catalogue des manuscripts scientifiques (Roger Mayer)

Philippe Bovier, De l'Asile à la Cité, Histoire de la Clinique Psychiatrique de Bel-Air et Transformation des domaines de la Folie (Roger Mayer)

Die Universität Zürich 1933-1983. Festschrift zur 150-Jahr-Feier (Carl Haffter)

Wolfgang Eckart, Johanna Geyer-Kordesch (Ed.), Heilberufe und Kranke im 17. und 18. Jahrhundert (Antoinette Stettler)

Robert G. Frank Jr., Harvey and the Oxford Physiologists. A Study of Scientific Ideas (Antoinette Stettler)

gelêrter der arzenîe, ouch apotêker. Festschrift zum 70. Geburtstag von Willem F. Daems (Gottfried Schramm)

Reinhard Gursch, Die Illustrationen Ernst Haeckels zur Abstammungs- und Entwicklungsgeschichte (Guido Sigron)

Thomas Haenel, Zur Geschichte der Psychiatrie. Gedanken zur allgemeinen und Basler Psychiatrie (Erwin H. Ackerknecht)

Hippocratica. Actes du Colloque hippocratique de Paris (septembre 1978) (Philippe Mudry)

Kurt Hofius, Rezeptjournale der Ratsapotheke Lehrte von 1899 und 1930

(François Ledermann)

Burkhard Hofmann, Kranker und Krankheit um 1500. Die Darstellung des Kranken im Zusammenhang mit den spätgotischen Bildnissen der Heiligen Elisabeth (Erwin H. Ackerknecht)

T.S.Hsu, The Human and Mammalian Cytogenetics. An Historical Perspective (Antoinette Stettler)

Wolfgang Jäger, Die Erfindung der Ophthalmoskopie (Huldrych M. Koelbing)

S. A. Jayawardene (Comp.), Reference Books for the Historian of Science (Felix Bloch)

Wolfgang G. Jilek, Indian Healing. Shamanic ceremonialism in the Pacific Northwest today (Erwin H. Ackerknecht)

Ramon Jordi Gonzàles, Cien años de vida farmacéutica barcelonesa (1830-1939) (François Ledermann)

Cornelius Keller, Die Geschichte der Radioaktivität unter besonderer Berücksichtigung der Transurane (Karl von Meyenn)

Henk J. Klasen, History of Free Skin Grafting, Knowledge or Empirism? (Antoinette Stettler)

Emil Kuhn-Schnyder, Lorenz Oken, 1779-1851 (Erwin H. Ackerknecht)

Henri de Lacaze-Duthiers, Léon Frederico, Correspondance 1878-1900 (Erwin H. Ackerknecht)

Laennec (1781-1826), Commémoration du bicentenaire de la naissance de Laennec (Roger Mayer)

Moritz Leisibach, Das Medizinisch-chirurgische Institut in Zürich 1782-1833 
Wolfgang Löchel, Die Zahnmedizin Rogers und der Rogerglossen (Guido Sigron)

José M. Lopez Pinero, Thomas F. Glick, Victor Navarro Brotbus, Eugenio Portela Marco, Diccionario Historico de la Ciencia Moderna en España (Erwin H. Ackerknecht)

Guglielmo Lützenkirchen, Saggio de bibliografia sulla scuola medica salernitana; Fabio Troncarelli, Catalogo della mostra fotografica (Urs Boschung)

Nicola G. Markoff-Gräflin, Als Arzt unterwegs. Erinnerungen und Begegnungen (Hans H. Walser)

Peter Morys, Medizin und Pharmazie in der Kosmologie Leonhard Thurneissers zum Thurn (1531-1596) (Antoinette Stettler)

Irmgard Müller, Die pflanzlichen Heilmittel bei Hildegard von Bingen (M.-L.Portmann)

Axel Heinrich Murken et al., Kind, Krankheit und Krankenhaus im Bilderbuch von 1900 bis 1982 (Erwin H. Ackerknecht)

Neue Beiträge zur Arzneimittelgeschichte. Festschrift für Wolfgang Schneider (Hans-Rudolf Fehlmann)

Nouvelle histoire de la psychiatrie (Ed. Jacques Postel et Claude Quetel)

(Erwin H. Ackerknecht)

Markus Oettli, Das Amt des Bezirksarztes («das Physikat») im Rahmen der Thurgauischen Sanitätsorganisation 1803-1869 (Erwin H. Ackerknecht)

Walter Pagel, Paracelsus. An Introduction to Philosophical Medicine in the Era of the Renaissance (Willem F. Daems)

Pharmazie und Geschichte. Festschrift für Günter Kallinich zum 65. Geburtstag (Hans-Rudolf Fehlmann)

K. A. Portele, Die Sammlung mazerierter Skelette und Knochen des Pathologisch-anatomischen Bundesmuseums, I. Teil (Urs Boschung)

Peter Potthoff, Der Tod im medizinischen Denken (Cecile Ernst)

Claus Priesner, H. Staudinger, H. Mark und K. H. Meyer. Thesen zur Größe und Struktur der Makromoleküle, Ursachen und Hintergründe eines akademischen Disputes (Eduard Preiswerk)

Hans Schadewaldt, Idiosynkrasie, Anaphylaxie, Allergie, Atopie - ein Beitrag zur Geschichte der Überempfindlichkeitskrankheiten (Carl Haffter)

Wulf Schiefenhövel und Dorothea Sich (Ed.), Die Geburt aus ethnomedizinischer Sicht (Erwin H. Ackerknecht)

Joseph Schiller, Na notion de l'organisation dans l'histoire de la biologie (Antoinette Stettler)

Francis Schiller, A Möbius Strip. Fin de siècle Neuropsychiatry and Paul Möbius (Erwin H. Ackerknecht)

Rudolf Schumacher, Die Musik in der Psychiatrie des 19. Jahrhunderts (Carl Haffter)

Seit über einem Jahrhundert ...: Verschüttete Alternativen in der Sozialpolitik (Antoinette Stettler)

Petrus Severinus og hans Idea medicinae philosophicae. En dansk paracelsist (Willem F. Daems)

H. M.Snelders, Het Gezelschap der hollandsche Scheikundigen. Amsterdamse chemici uit het einde van de achttiende eeuw (Willem F. Daems)

K. Steinbereitner, H. Bergmann (Ed.), 25 Jahre Anaesthesiologie und Intensivtherapie in Österreich (W. Dimai) 
Léon Szyfman, Jean-Baptiste Lamarck et son époque (Erwin H. Ackerknecht)

Pierre L. Thillaud, Les Maladies et la Médecine en Pays Basque Nord à la fin de

Nelly Tsouyopoulos, Andreas Röschlaub und die Romantische Medizin (Willem F.Daems)

J. A. Veiga-Pires, Ronald Grainger (Ed.) Pioneers in Angiography (Erwin H. Ackerknecht)

Almuth Weidmann, Die Arzneiversorgung der Armen zu Beginn der Industrialisierung im deutschen Sprachgebiet, besonders in Hamburg (François Ledermann)

Nachrufe:

Jean Starobinski, Georges de Morsier (1894-1982)

Martin Vosseler, Adolf Portmann (1897-1982)

Hans H. Walser, Oscar Forel (1891-1982)

Mitteilungen

Errata 


\section{Inhalt \\ Sommaire}

Gerhard Rudolph, Herkunft und Schicksal des Humor aqueus 24.1

Loris Premuda, Die Medizin in der Sicht eines italienischen Denkers der Aufklärung 249

Ingrid Rohland und Gundolf Keil, Randnotizen zum «Schüpfheimer Kodex〉 257

Huldrych M. Koelbing, Bemerkungen zu Ingrid Rohlands und Gundolf Keils "Randnotizen zum «Schüpfheimer Kodex»»

W. E. Knowles Middleton, The Sun glasses used by H.B. de Saussure 277

Heinrich Stamm, 50 Jahre medizinische Forschung in Waldenburg 281

\section{Buchbesprechungen}

Nouvelle histoire de la psychiatrie (Ed. Jacques Postel et Claude Quetel)

(Erwin H. Ackerknecht)

Wulf Schiefenhövel und Dorothea Sich (Ed.), Die Geburt aus ethnomedizinischer Sicht (Erwin H. Ackerknecht)

Wolfgang G. Jilek, Indian Healing. Shamanic ceremonialism in the Pacific Northwest today (Erwin H. Ackerknecht)

José M. Lopez Pinero, Thomas F. Glick, Victor Navarro Brotbus, Eugenio Portela

Marco, Diccionario Historico de la Ciencia Moderna en España

(Erwin H. Ackerknecht)

Burkhard Hofmann, Kranker und Krankheit um 1500. Die Darstellung des Kranken im Zusammenhang mit den spätgotischen Bildnissen der Heiligen Elisabeth

(Erwin H. Ackerknecht)

Nicola G. Markhoff-Gräflin, Als Arzt unterwegs. Erinnerungen und Begegnungen (Hans H. Walser)

Hans Schadewaldt, Idiosynkrasie, Anaphylaxie, Allergie, Atopie-ein Beitrag zur Geschichte der Überempfindlichkeitskrankheiten (Carl Haffter)

Die Universität Zürich 1933-1983. Festschrift zur 150-Jahr-Feier (Carl Haffter)

Moritz Leisibach, Das Medizinisch-chirurgische Institut in Zürich 1782-1833 (Carl Haffter)

Rudolf Schumacher, Die Musik in der Psychiatrie des 19. Jahrhunderts (Carl Haffter)

Beiträge zur Schwäbischen Literatur- und Geistesgeschichte, Bd.2/1982 (Carl Haffter)

Hippocratica. Actes du Colloque hippocratique de Paris (septembre 1978) (Philippe Mudry)

Wolfgang Eckart, Johanna Geyer-Kordesch (Ed.), Heilberufe und Kranke im 17. und 18. Jahrhundert (Antoinette Stettler)

Joseph Schiller, La notion de l'organisation dans l'histoire de la biologie

(Antoinette Stettler)

R. Toellner (Ed.), Humanismus und Aufklärung (Antoinette Stettler)

(Antoinette Stettler)

Robert G. Frank Jr., Harvey and the Oxford Physiologists. A Study of Scientific Ideas (Antoinette Stettler) 
Peter Morys, Medizin und Pharmazie in der Kosmologie Leonhard Thurneissers zum Thurn (1531-1596) (Antoinette Stettler)

Seit über einem Jahrhundert ...: Verschüttete Alternativen in der Sozialpolitik (Antoinette Stettler)

Henk J. Klasen, History of Free Skin Grafting, Knowledge or Empiricism? (Antoinette Stettler)

Jean-Charles Sournia, Histoire et médecine (Roger Mayer)

Laennec (1781-1826), Commémoration du bicentenaire de la naissance de Laennec (Roger Mayer)

L. Boulle, M. D. Grmek, C. Lupovici, J.Samion-Contet, Laennec. Catalogue des manuscrits scientifiques (Roger Mayer)

Pierre L. Thillaud, Les Maladies et la Médecine en Pays Basque Nord à la fin de l'Ancien Régime (1690-1789) (Roger Mayer)

Philippe Bovier, De l'Asile à la Cité. Histoire de la Clinique Psychiatrique de Bel-Air et Transformation des domaines de la Folie (1900-1975) (Roger Mayer)

K. A. Portele, Die Sammlung mazerierter Skelette und Knochen des Pathologischanatomischen Bundesmuseums, I. Teil (Urs Boschung)

Guglielmo Lützenkirchen, Saggio de bibliografia sulla scuola medica salernitana; Fabio Troncarelli, Catalogo della mostra fotografica (Urs Boschung)

K. Steinbereithner, H. Bergmann (Ed.), 25 Jahre Anaesthesiologie und Intensivtherapie in Österreich (W. Dimai)

Wolfgang Löchel, Die Zahnmedizin Rogers und der Rogerglossen (Guido Sigron)

Reinhard Gursch, Die Illustrationen Ernst Haeckels zur Abstammungs- und Entwicklungsgeschichte (Guido Sigron)

Peter Potthoff, Der Tod im medizinischen Denken (Cecile Ernst)

Medizin im mittelalterlichen Abendland (Herausgeber: Gerhard Baader und Gundolf Keil) (Felix Bloch)

gelêrter der arzenîe, ouch apotêker. Festschrift zum 70. Geburtstag von Willem F. Daems (Gottfried Schramm)

H. A. M.Snelders, Het Gezelschap der hollandsche Scheikundigen. Amsterdamse chemici uit het einde van de achttiende eeuw (Willem F. Daems)

Petrus Severinus og hans Idea medicinae philosophicae. En dansk paracelsist (Willem F. Daems)

Richard Löw, Pflanzenchemie zwischen Lavoisier und Liebig (Willem F. Daems)

Walter Pagel, Paracelsus. An Introduction to Philosophical Medicine in the Era of the Renaissance (Willem F. Daems)

Nelly Tsouyopoulos, Andreas Röschlaub und die Romantische Medizin (Willem F.Daems)

Pharmazie und Geschichte. Festschrift für Günter Kallinich zum 65. Geburtstag (Hans Rudolf Fehlmann)

Neue Beiträge zur Arneimittelgeschichte. Festschrift für Wolfgang Schneider (Hans-Rudolf Fehlmann)

Irmgard Müller, Die pflanzlichen Heilmittel bei Hildegard von Bingen (M.-L. Portmann)

Kurt Hofius, Rezeptjournale der Ratsapotheke Lehrte von 1899 und 1930 (François Ledermann)

Almuth Weidmann, Die Arzneiversorgung der Armen zu Beginn der Industrialisierung im deutschen Sprachgebiet, besonders in Hamburg (François Ledermann)

Ramon Jordi Gonzàlez, Cien años de vida farmacéutica barcelonesa (1830-1939) (François Ledermann) 
Cornelius Keller, Die Geschichte der Radioaktivität, unter besonderer Berücksichtigung der Transurane (Karl von Meyenn)

Claus Priesner, H.Staudinger, H. Mark und K.H. Meyer. Thesen zur Größe und

Struktur der Makromoleküle, Ursachen und Hintergründe eines akademischen Disputes (Eduard Preiswerk)

Nachrufe:

Jean Starobinski, Georges de Morsier (1894-1982)

Martin Vosseler, Adolf Portmann (1897-1982)

Hans H. Walser, Oscar Forel (1891-1982)

Mitteilungen

Errata

Inhalt des Jahrgangs 40 / Sommaire du volume 40 